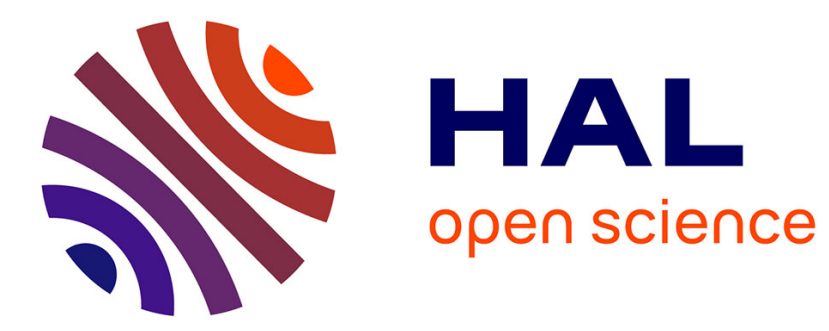

\title{
Hypoxia and non alcoholic fatty liver disease
}

Christopher D Byrne

\section{- To cite this version:}

Christopher D Byrne. Hypoxia and non alcoholic fatty liver disease. Clinical Science, 2009, 118 (6), pp.397-400. 10.1042/CS20090565 . hal-00546867

\section{HAL Id: hal-00546867 https://hal.science/hal-00546867}

Submitted on 15 Dec 2010

HAL is a multi-disciplinary open access archive for the deposit and dissemination of scientific research documents, whether they are published or not. The documents may come from teaching and research institutions in France or abroad, or from public or private research centers.
L'archive ouverte pluridisciplinaire HAL, est destinée au dépôt et à la diffusion de documents scientifiques de niveau recherche, publiés ou non, émanant des établissements d'enseignement et de recherche français ou étrangers, des laboratoires publics ou privés. 


\title{
Hypoxia and non alcoholic fatty liver disease
}

Endocrinology \& Metabolism

The Institute of Developmental Sciences (IDS Building) (University of Southampton), MP 887 Southampton General Hospital Tremona Rd Southampton SO16 6YD

Christopher D Byrne

\begin{abstract}
Non alcoholic fatty liver disease (NAFLD) represents a spectrum of fatty liver diseases associated with increased risk of type 2 diabetes and cardiovascular disease. The spectrum of fatty liver diseases, comprises simple steatosis, steatosis with inflammation (i.e. non alcoholic steatohepatitis or NASH), fatty liver disease with inflammation and fibrosis (severe NASH) and cirrhosis. The molecular mechanisms contributing to NASH are the subject of considerable investigation, as a better understanding of the pathogenesis of NASH will lead to novel therapies for a condition that hitherto remains difficult to treat. In this edition of the Journal Piquet et al have investigated the effects of hypoxia in the PTEN deficient mouse, a mouse model that develops NAFLD. Piquet et al show that a short period ( 7 days) of exposure to hypoxia aggravates the NAFLD phenotype, causing changes in the liver that are in keeping with NASH, with increased lipogenesis and inflammation.
\end{abstract}

Risk factors for non alcoholic fatty liver disease (NAFLD) continue to increase with the epidemic of obesity and type 2 diabetes. NAFLD is not a single disease but represents a spectrum of fatty liver-related diseases, extending from simple steatosis to steatosis with ballooned hepatoytes and lobular inflammation [non alcoholic steatohepatitis (NASH)], to extensive hepatic fibrosis with regenerating nodules (cirrhosis). In some patients the liver condition may lead to the development of hepatocellular carcinoma. For researchers, clinicians and their patients, the key question is why do some people develop only simple steatosis without progression of the liver disease, whilst others develop a progressive liver condition with NASH developing and worsening over time to cirrhosis (for review see [2].

Piguet et al have investigated the effect of hypoxia on NAFLD. Specifically the authors have tested the effect of relative hypoxia ( $10 \%$ inspired oxygen) in a mouse model that is genetically susceptible to developing NAFLD; to test the hypothesis that relative hypoxia worsens progression of NAFLD to NASH. Using mice with a specific hepatocellular deficiency in the phosphatase and tensin homologue gene (PTEN) gene, a tumour suppressor that is known to affect the insulin signaling pathway [13-15]; the investigators have subjected the animals to 7 days of $10 \%$ inspired oxygen tension, compared to 7 days of $21 \%$ inspired oxygen in the control PTEN deficient animals. To prove that the animals were hypoxic, Piguet at showed that there was a $10 \%$ increase in haematocrit. Affected animals were more insulin resistant, lighter in weight, hypertriglycerdaemic, glucose intolerant and had a marked increase in Kleiner score, a histopathogical scoring system used to quantitative severity of NAFLD, $(8.3$ v. $2.3, \mathrm{p}<0.01)$ in keeping with NASH. In investigating the pathogenesis of hypoxia-induced NASH, Piquet et al show that 
expression of key genes in the hepatic lipogenesis pathway were increased, whereas expression of a marker of beta oxidation (CPT-1) was decreased. The substrate proteins for the PTEN enyzme are phosphatidylinositol, 3,4 biphosphate and phosphatidylinositol $3,4,5$ triphosphate, and the physiological function of PTEN is to dephosphorylate the second messengers generated by the activation of phosphatidylinositol -3- kinase (PI3K) thereby downregulating or terminating insulin signal downstream of PI3 kinase [13]. Therefore the authors investigated the insulin signaling pathway to explore the effects of hypoxia on NAFLD.

Hypoxia induced an increase in phosphorylation status of the $\mathrm{S} 6$ ribosomal protein. The S6 ribosomal protein belongs to the PI3 kinase/Akt/mTOR signaling pathway and is phosphorylated by p70 S6 kinase when this pathway is activated. It has been shown previously that a negative feedback look operates from p70 S6 kinase to the upstream IRS-PI3K-PDK1-Akt insulin signaling pathway suggesting a mechanism for the development of insulin resistance [3;11] and such a mechanism could operate in the model generated by Piguet et al.

There is, however, still uncertainty as to the precise role of PTEN activity in regulating insulin sensitivity in insulin responsive tissues. Over expression of PTEN has been shown to have inhibitory effects on insulin signalling including decreased Akt activation and glut 4 translocation to the cell membrane [7;8]. Over expression of PTEN in muscle from obese $\mathrm{Fa} / \mathrm{Fa}$ Zucker rats had been shown to contribute to muscle insulin resistance in these animals [5]. In contrast, downregulation of PTEN has the opposite effect with increased glucose uptake in fat and muscle in response to insulin [10]. In mice, liver specific deletion of PTEN, has been shown previously to increase insulin sensitivity yet paradoxically causes NAFLD and hepatocellular cancer [4;9]. The mechanism for this paradox is yet to be clarified but there has been a number of hypotheses suggested, some of which highlight the lack of the negative regulation on the insulin signaling pathways by PTEN. In the PTEN deficient mice, there is increased synthesis and storage of triglyceride in hepatocytes perhaps due to upregulation of the PI3K/Akt activity [4;9] and as a consequence of the lack of PTEN activity, there is increased hepatocyte fatty acid uptake, increased fatty acid synthesis and increased esterification of fatty acid to triglyceride.

Absent expression of liver-specific PTEN is associated with hepatic steatosis, inflammation, fibrosis and even tumour [15]. In one report, PTEN- deficient mice were shown to have biochemical and histological evidence of NASH, with fibrosis occurring at 40 weeks age [15]. The mechanism for NASH in this animal model has been reported to be due to increased expression of the PPAR- $\gamma$, SREBP-1c and the downstream genes including Akt, Foxol resulting in increased lipogenesis, inflammation and fibrosis [15].The reason for the increased fat deposition in the liver could be due partly to increased expression of SREBP-1c and SREBP-1c is a key transcription factor for lipogenesis with increased acetyl CoA carboxylase, fatty acid synthetase and sterol-CoA desaturase-1 enzyme activities, all these acting synergistically to promote fatty acid synthesis. Piguet et al show very similar effects in much younger 8 week old female PTEN deficient mice, exposed to hypoxia for 7 days, suggesting that relative hypoxia 
accelerates many of the changes that have been observed in older PTEN deficient mice developing NASH.

As a consequence of increased PPAR- $\gamma$ expression, there is also a secondary induction of key enzymes involve in mitochondrial $\beta$ oxidation and interestingly Piguet et al show a hypoxia-induced increase in HIF 2 alpha, with translocation of HIF 2 alpha to the nucleus, that has been shown to inhibit $\beta$ oxidation. Whether this effect alters the known increase in reactive oxygen species that occurs in PTEN deficient mice is uncertain. PTEN deficient mice show an increase in fat oxidation with a marked increase in the generation of oxidative free radicals, leading to inflammation and fibrosis, via activation of the NF$\kappa \mathrm{B}$ pathway $[6 ; 16]$. It has been shown that there is a seven fold increase in the hepatic concentration of hydrogen peroxide in PTEN deficient mice compared to the wild type [15] and in their hypoxia model, Piguet et al suggest that the increase in cytochrome P450 $2 \mathrm{E} 1$, that is known to increase oxidative stress, may contribute to the increase in inflammation observed in their model. Whether any change in P450 2E1 activity occurs as a consequence or cause of insulin resistance is not known. Moreover, the role of inflammation in vivo may influence the activity of PTEN to further influence insulin action. In insulin resistant subjects, there is increased plasma free fatty acid concentration with increase delivery and, uptake of free fatty acid by the hepatocytes. This results in the activation of the mTOR protein, which in turn leads to activation of NF- $\kappa B$ [14]. Since both mTOR and NF- $\kappa$ B exist as a complex, there is a subsequent dissociation and, then translocation of NF- $\kappa B$ into the nucleus where it downregulates the expression of PTEN at the level of transcription. The precise mechanism of how NF- $\kappa B$ transcriptionally down regulates PTEN is not fully understood but could be through the sequestration of the $\mathrm{CBP} / \mathrm{p} 300$, the transcriptional activator for PTEN [12].

Is hypoxia alone sufficient to cause NAFLD? To date the evidence suggests that hypoxia aggravates development of NAFLD only in susceptible animals, with worsening of NAFLD to a NASH-like phenotype. The last year has shown important advances in our understanding of factors affecting aetiology and pathogenesis of NASH, such as the potential for the influence of early development to prime development of NASH [1]. Given the important clinical need to elucidate the mechanisms contributing to NASH and to subsequently develop treatments for NASH, the work of Piguet and colleagues adds to our understanding of the potential interaction of exogenous fatty acids, PTEN deficiency and hypoxia in the pathogenesis of NASH (summarized in figure 1). The challenge is now on to ascertain whether similar changes occur in people at risk of developing NAFLD.

\section{Conflict of interest statement}

The author has no conflicts of interests

\section{Legend}

Figure 1 Schematic figure illustrating the potential role of exogenous fatty acid, PTEN deficiency and hypoxia in the development of NASH 
Reference List

1. Bruce,K.D., Cagampang,F.R., Argenton,M. et al. (2009) Maternal high-fat feeding primes steatohepatitis in adult mice offspring, involving mitochondrial dysfunction and altered lipogenesis gene expression. Hepatology 2009 Aug 5 ePub.

2. Byrne,C.D., Olufadi,R., Bruce,K.D., Cagampang,F.R. and Ahmed,M.H. (2009) Metabolic disturbances in non-alcoholic fatty liver disease. Clin.Sci.(Lond) 116, 539-564.

3. Harrington,L.S., Findlay,G.M., Gray,A. et al. (2004) The TSC1-2 tumor suppressor controls insulin-PI3K signaling via regulation of IRS proteins. J.Cell Biol. 166, 213-223.

4. Horie,Y., Suzuki,A., Kataoka,E. et al. (2004) Hepatocyte-specific Pten deficiency results in steatohepatitis and hepatocellular carcinomas. J.Clin.Invest 113, 17741783.

5. Lo,Y.T., Tsao,C.J., Liu,I.M., Liou,S.S. and Cheng,J.T. (2004) Increase of PTEN gene expression in insulin resistance. Horm.Metab Res. 36, 662-666.

6. Marcus,S.L., Miyata,K.S., Zhang,B., Subramani,S., Rachubinski,R.A. and Capone,J.P. (1993) Diverse peroxisome proliferator-activated receptors bind to the peroxisome proliferator-responsive elements of the rat hydratase/dehydrogenase and fatty acyl-CoA oxidase genes but differentially induce expression. Proc.Natl.Acad.Sci.U.S.A 90, 5723-5727.

7. Nakashima,N., Sharma,P.M., Imamura,T., Bookstein,R. and Olefsky,J.M. (2000) The tumor suppressor PTEN negatively regulates insulin signaling in 3T3-L1 adipocytes. J.Biol.Chem. 275, 12889-12895.

8. Ono,H., Katagiri,H., Funaki,M. et al. (2001) Regulation of phosphoinositide metabolism, Akt phosphorylation, and glucose transport by PTEN (phosphatase and tensin homolog deleted on chromosome 10) in 3T3-L1 adipocytes. Mol.Endocrinol. $15,1411-1422$

9. Stiles,B., Wang,Y., Stahl,A. et al. (2004) Liver-specific deletion of negative regulator Pten results in fatty liver and insulin hypersensitivity [corrected]. Proc.Natl.Acad.Sci.U.S.A 101, 2082-2087.

10. Tang,X., Powelka,A.M., Soriano,N.A., Czech,M.P. and Guilherme,A. (2005) PTEN, but not SHIP2, suppresses insulin signaling through the phosphatidylinositol 3kinase/Akt pathway in 3T3-L1 adipocytes. J.Biol.Chem. 280, 22523-22529. 
11. Tremblay,F. and Marette,A. (2001) Amino acid and insulin signaling via the mTOR/p70 S6 kinase pathway. A negative feedback mechanism leading to insulin resistance in skeletal muscle cells. J.Biol.Chem. 276, 38052-38060.

12. Vasudevan,K.M., Gurumurthy,S. and Rangnekar,V.M. (2004) Suppression of PTEN expression by NF-kappa B prevents apoptosis. Mol.Cell Biol. 24, 1007-1021.

13. Vinciguerra,M. and Foti,M. (2006) PTEN and SHIP2 phosphoinositide phosphatases as negative regulators of insulin signalling. Arch.Physiol Biochem. 112, 89-104.

14. Vinciguerra,M., Veyrat-Durebex,C., Moukil,M.A., Rubbia-Brandt,L., RohnerJeanrenaud,F. and Foti,M. (2008) PTEN down-regulation by unsaturated fatty acids triggers hepatic steatosis via an NF-kappaBp65/mTOR-dependent mechanism. Gastroenterology 134, 268-280.

15. Watanabe,S., Horie,Y., Kataoka,E. et al. (2007) Non-alcoholic steatohepatitis and hepatocellular carcinoma: lessons from hepatocyte-specific phosphatase and tensin homolog (PTEN)-deficient mice. J.Gastroenterol.Hepatol. 22 Suppl 1, S96-S100.

16. Yu,S., Matsusue,K., Kashireddy,P. et al. (2003) Adipocyte-specific gene expression and adipogenic steatosis in the mouse liver due to peroxisome proliferator-activated receptor gamma1 (PPARgamma1) overexpression. J.Biol.Chem. 278, 498-505. 


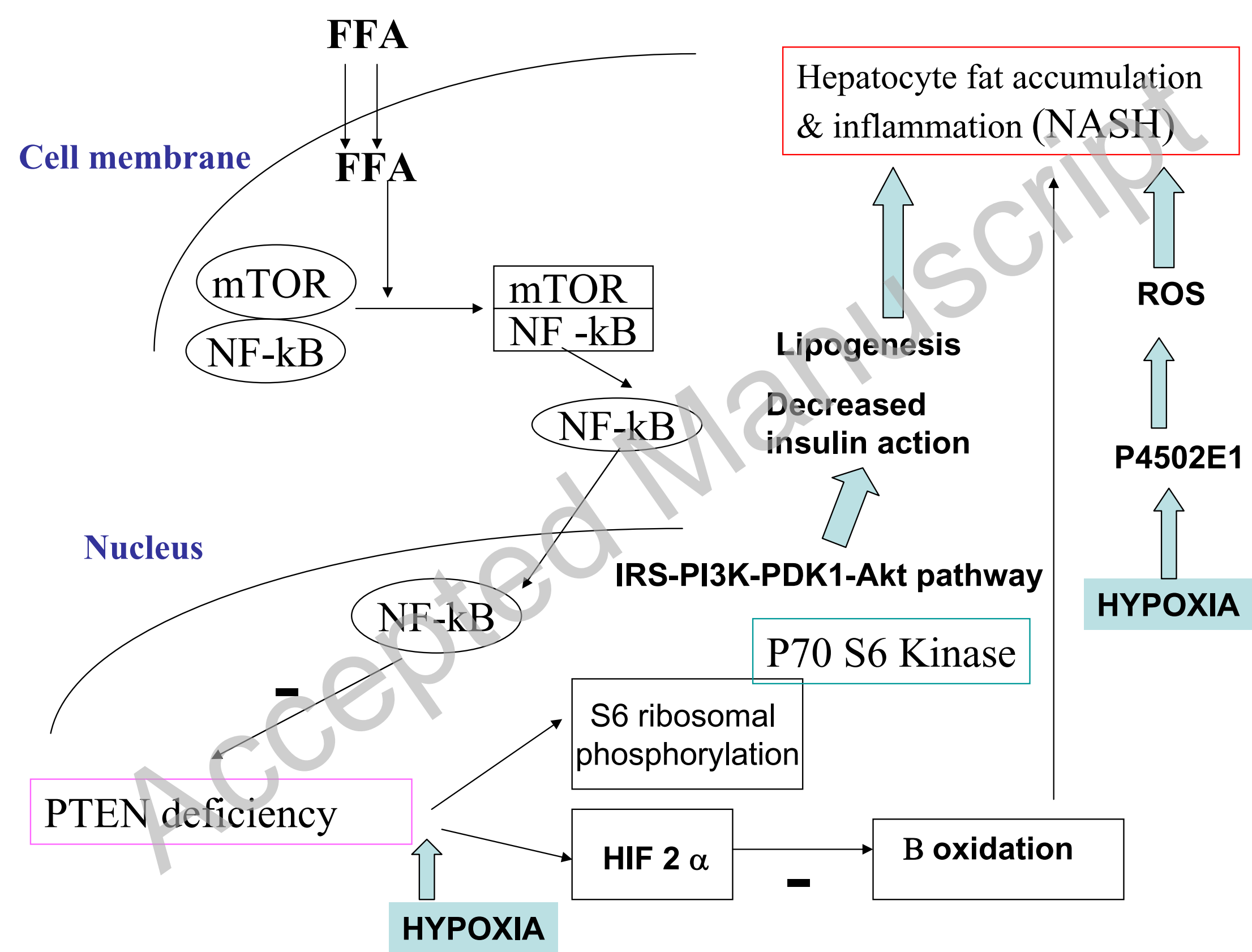

